\title{
Antineutrophil cytoplasmic antibodies and their association with clinical outcomes in hospitalized COVID-19 patients
}

\author{
Kamran Kadkhoda $\mathbb{D}^{1,2 \bowtie}$ and Kristen Laurita ${ }^{1}$ \\ (c) The Author(s) 2021 \\ Cell Death Discovery (2021)7:277; https://doi.org/10.1038/s41420-021-00671-1
}

As indubitably the most catastrophic pandemic of the $21^{\text {st }}$ century so far, coronavirus disease 2019 (COVID-19) has shown many faces. This ranges from direct viral cytopathic effect to triggering immunopathological mechanisms through which COVID-19 can further exert tissue damage. One of the important features is the elevated neutrophil-to-lymphocyte-count ratio, especially during moderate and severe COVID-19 and the elevated circulating neutrophil extracellular traps (NETs) [1-5]. NETs are typically studded with several proteins, including a variety of neutrophils' enzymes. NETs, in turn, can activate complement pathways further causing tissue destruction and vasculitis. Antineutrophil cytoplasmic antibodies (ANCAs) are a type of autoantibodies that are typically directed against neutrophils' cationic enzymes such as proteinase 3 (PR3), myeloperoxidase (MPO), elastase, lactoferrin, cathepsin G, bacterial permeability-increasing peptide, and calprotectin. They are involved in the pathomechanism of small-tomedium-sized vessel vasculitides. ANCAs can be detected by indirect immunofluorescence assay (IFA) and are named based on the staining pattern. If the neutrophil cytoplasm is almost evenly stained but the nucleus is not, it is called cytoplasmic ANCA (CANCA), whereas when only the nucleus is stained but the cytoplasm is not, it is called perinuclear ANCA (P-ANCA). Although not universal, PR3 is associated with the former and MPO with the latter. C- or P-ANCAs can be by-products of the aforementioned NET-formation mechanism [1]. Although ANCAs are known to play a role in vasculitides generally in the context of autoimmune diseases, their generation can also be elicited by certain infections such as Staphylococcus aureus [4].

Given the known pathophysiology of COVID-19 so far, we set out to investigate the prevalence of ANCAs among 100 randomly selected hospitalized patients with confirmed COVID-19 diagnoses in 2020. Blood specimens were drawn from these patients at the Cleveland Clinic hospitals and stored at the biorepository for future studies. Our study was approved by the institutional review board. There were two groups of 50 patients: moderate and severe, the latter was defined as patients with any of intensive care unit (ICU) admission, mechanical ventilation, extracorporeal membrane oxygenation (ECMO), or death, whereas the moderate cases did not meet any of the above-mentioned criteria. Samples were chosen randomly by the institutional biorepository staff based on the requested criteria and then were blinded to the investigators. Samples were collected from 9 to 28 days (median 19.5) post onset of signs and symptoms from patients with a median age of 59 years (range: 21-91). In total, 51 patients were male and the rest were female. Overall, $48 \%$ had ICU admission, $31 \%$ had mechanical ventilation, none received ECMO, and $15 \%$ died. All samples were screened for ANCAs by IFA using ethanolfixed slides. Given the almost pervasive fluorescence activity among the screen slides, the samples were all also examined for ANCAs using formalin-fixed slides as the confirmatory test. All samples were also tested for anti-PR3 and anti-MPO antibodies using a random-access platform based on multiplex-fluorescence immunoassay (MFIA) principle. The three tests mentioned above are routinely performed in our laboratory.

Overall, $57 \%$ of sera tested confirmed positive for ANCAs, of which $72 \%$ and $28 \%$ were confirmed C-ANCA and P-ANCA, respectively. Only one sample tested positive for anti-MPO antibody; three other samples with matching ANCA results had antibody-index values of $0.4-0.6$ range (cutoff: 1.0 ), suggesting some had weak reactivity below the cutoff. Of 15 patients who died, $17.5 \%$ had confirmed ANCA results compared with $11.6 \%$ of survivors with no confirmed ANCA result, though this did not reach statistical significance. Furthermore, $63.4 \%$ of severe cases had confirmed C-ANCA results compared with $34.9 \%$ of patients with no confirmed ANCAs (OR: 1.81, 95\% Cl: 0.86-3.75; $P=0.13$, Fisher exact). There was no association between age and sex with ANCA positivity. A subtle, but nonsignificant trend, was noticed with P-ANCA positivity with increasing age. Most interestingly, there was a strong association between confirmed C-ANCA status with ICU admission (OR: $3.250,95 \% \mathrm{Cl}: 1.31-7.60, P=0.0087$ ) in contrast to the confirmed P-ANCA status, whereas this association was marginal for mechanical ventilation (OR: 3; $95 \% \mathrm{Cl}$ : 1.08-8.67, $P=0.05$ ) (Table 1).

Our finding of such high prevalence of ANCAs among hospitalized patients was unexpected and very exciting. To the best of our knowledge, this is the first time that such study is done in these patients, especially using IFA for ANCAs. As mentioned earlier, there are a number of neutrophil enzymes that can trigger ANCAs, but with the exception of PR3 and MPO, they are not tested for using current immunoassays such as MFIA that was used here. In fact, when NETs are produced, they are decorated with these enzymes, so under natural circumstances they can destroy microorganisms such as bacteria that are stuck in these NETs. Since this is a local reaction, the nearby endothelial cells are damaged not only directly by these detrimental enzymes but also through triggering immunothrombosis by these enzymes. The

\footnotetext{
${ }^{1}$ Immunopathology Laboratory, Robert J. Tomsich Pathology \& Laboratory Medicine Institute, Cleveland Clinic, Cleveland, OH, USA. ${ }^{2}$ Cleveland Clinic Lerner College of Medicine, Case Western Reserve University, Cleveland, OH, USA. ${ }^{\circledR}$ email: kadkhok@ccf.org
}

Received: 18 August 2021 Revised: 8 September 2021 Accepted: 23 September 2021

Published online: 05 October 2021 
Table 1. Antineutrophil cytoplasmic antibodies and their association with clinical outcomes in hospitalized COVID-19 patients.

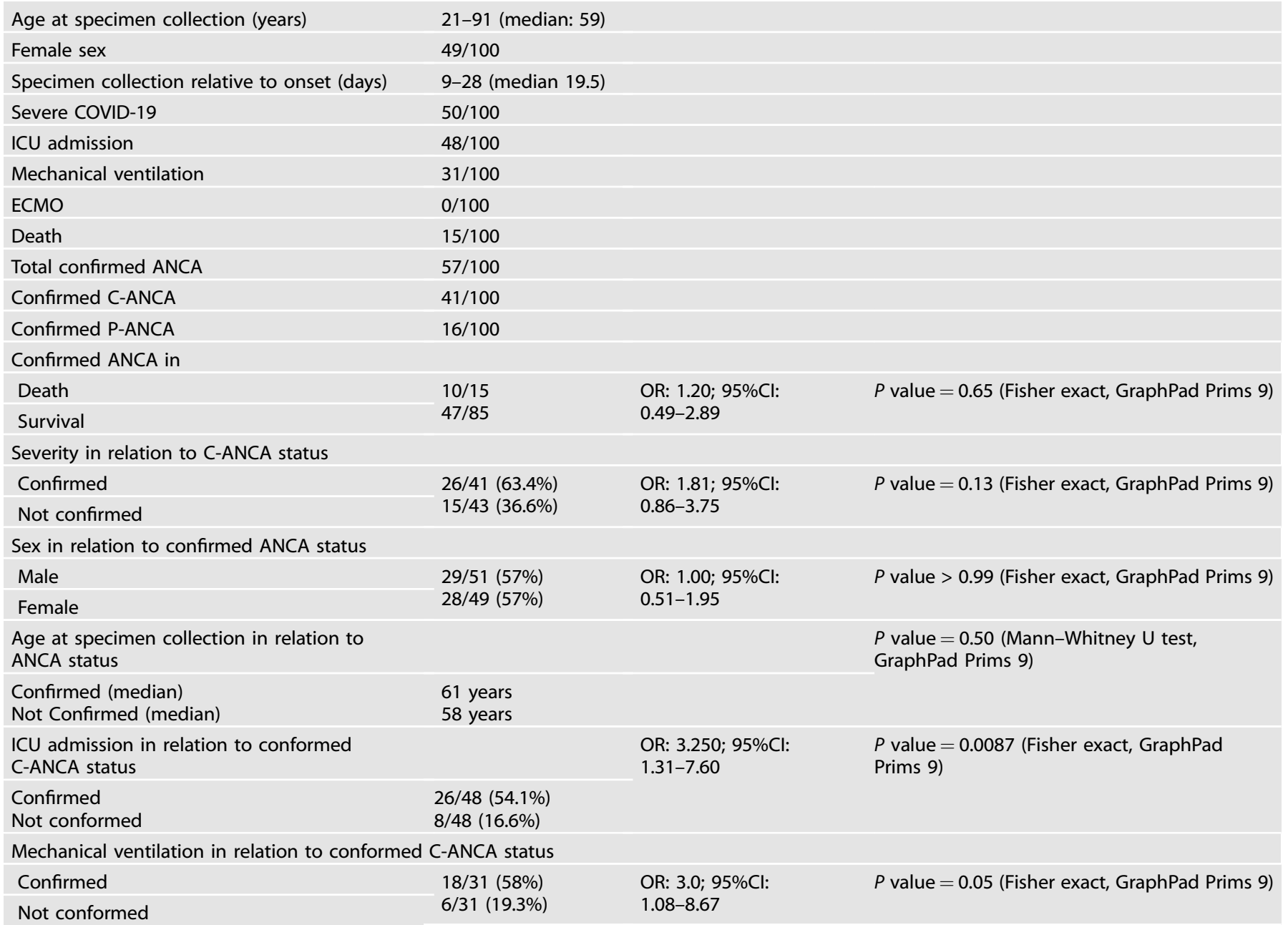

latter can be particularly problematic in tissues with large vasculature such as lungs, leading to pulmonary embolism. Through production of $\mathrm{C} 5 \mathrm{a}$, complement activation due to the presence of NETs attracts more neutrophils to the site, further exacerbating the situation.

Since we did not have samples available before the onset of COVID-19 or after hospital discharge, we could not follow up on the ANCA status over time. It is important to highlight that both ANCA and MPO/PR3 tests detected IgG antibodies, thus, we could not rule out the presence of $\operatorname{lgM}$ antibodies, given the short period after COVID-19 onset, hence little time for a class-switched autoimmune response to fully develop. This means the several screen-positive-only sera would have possibly been confirmed had the study included specimens drawn several weeks or months after onset. Of note, that corticosteroids are used for managing COVID-19 patients on mechanical ventilation or in the ICU, and are also used routinely for managing patients with ANCA-associated vasculitides, points to a possible shared pathomechanism. This study calls for longitudinal follow-ups in patients with a history of confirmed COVID-19 diagnosis who have been experiencing autoimmune phenomena suggestive of vasculitis. Routinely investigating ANCA among hospitalized COVID-19 patients, especially shortly after admission, may serve as a biomarker to predict worse outcomes. This notion needs substantiating through longitudinal studies.

\section{DATA AVAILABILITY}

Data are available to the journal and the publisher upon request.

\section{REFERENCES}

1. Ackermann M, Anders HJ, Bilyy R, Bowlin GL, Daniel C.De Lorenzo R,et al.Patients with COVID-19: in the dark-NETs of neutrophils.Cell Death Differ. 2021;1-15. https://doi.org/10.1038/s41418-021-00805-z

2. Chang SE, Feng A, Meng W, Apostolidis SA, Mack E, Artandi M et al. New-Onset lgG Autoantibodies in Hospitalized Patients with COVID-19. medRxiv, https://doi.org/ 10.1101/2021.01.27.21250559 (2021).

3. Izci Duran T, Turkmen E, Dilek M, Sayarlioglu $H$, Arik N. ANCA-associated vasculitis after COVID-19. Rheumatol Int. 2021;41:1523-9. https://doi.org/10.1007/s00296021-04914-3

4. Nakazawa D, Masuda S, Tomaru U, Ishizu A. Pathogenesis and therapeutic interventions for ANCA-associated vasculitis. Nat Rev Rheumatol. 2019;15:91-101. https://doi.org/10.1038/s41584-018-0145-y

5. Narasaraju T, Tang BM, Herrmann M, Muller S, Chow VTK, Radic M. Neutrophilia and NETopathy as key pathologic drivers of progressive lung impairment in patients with COVID-19. Front Pharm. 2020;11:870 https://doi.org/10.3389/ fphar.2020.00870

\section{ACKNOWLEDGEMENTS}

The authors would like to thank Dr. Jennifer Ko, Alexis Plaga, Greg Strnad, and Lisa Olson for their support. 


\section{AUTHOR CONTRIBUTIONS}

KK performed literature review, conceptualization, experiment designs, data analysis, and writing, obtained applicable approvals and funding; $\mathrm{KL}$ performed the experiments.

\section{FUNDING}

This project was supported by the Cleveland Clinic Foundation Pathology \& Laboratory Medicine Institute internal research funds.

\section{COMPETING INTERESTS}

The authors declare no competing interests.

\section{ADDITIONAL INFORMATION}

Supplementary information The online version contains supplementary material available at https://doi.org/10.1038/s41420-021-00671-1.

Correspondence and requests for materials should be addressed to Kamran Kadkhoda.
Reprints and permission information is available at http://www.nature.com/ reprints

Publisher's note Springer Nature remains neutral with regard to jurisdictional claims in published maps and institutional affiliations.

Open Access This article is licensed under a Creative Commons Attribution 4.0 International License, which permits use, sharing, adaptation, distribution and reproduction in any medium or format, as long as you give appropriate credit to the original author(s) and the source, provide a link to the Creative Commons license, and indicate if changes were made. The images or other third party material in this article are included in the article's Creative Commons license, unless indicated otherwise in a credit line to the material. If material is not included in the article's Creative Commons license and your intended use is not permitted by statutory regulation or exceeds the permitted use, you will need to obtain permission directly from the copyright holder. To view a copy of this license, visit http://creativecommons. org/licenses/by/4.0/.

(c) The Author(s) 2021 